\title{
ANTONOVSKY'S SENSE OF COHERENCE AND JOB SATISFACTION: Meta-Analyses of South African data
}

\section{Authors: \\ Deo J.W. Strümpfer \\ Gideon P. de Bruin ${ }^{2}$}

\section{Affiliations:}

${ }^{1}$ Department of

Psychology, University of Johannesburg, South

Africa

${ }^{2}$ Department of Human Resource Management, University of Johannesburg, South Africa

\section{Correspondence to:} Gideon P. de Bruin

e-mail:

deondb@uj.ac.za

\section{Postal address:}

Department of Industrial Psychology and People Management, University of Johannesburg, Box 254, Auckland Park, Gauteng, 2006, South Africa

\section{Keywords:}

job satisfaction; sense of coherence; meta-analysis; stress management; job performance

\section{Dates:}

Received: 17 Nov. 2008 Accepted: 15 July 2009 Published: 11 Sept. 2009

How to cite this article: Strümpfer, D.J.W., \& De Bruin, G.P. (2009). Antonovsky's sense of coherence and job satisfaction: Meta-analyses of South African data. SA Journal of Industrial Psychology/SA Tydskrif vir Bedryfsielkunde, 35(1), Art. \#767, 3 pages. DOI: 10.4102/ sajip.v35i1.767

This article is available at:

http://www.sajip.co.za

(C) 2009. The Authors. Licensee: OpenJournals Publishing. This work is licensed under the Creative Commons Attribution License.

\section{ABSTRACT}

Data on the relationship between sense of coherence (SOC) and job satisfaction (JS) were collected from published sources, unpublished theses and unpublished reports. Twenty-four data sets using the SOC long form and 10 sets using the short form were found. Neither the form of SOC nor the type of JS measure moderated the relation. SOC accounted for approximately $18 \%$ of the variance in JS. Approximately $10 \%$ of the between-study variance was due to real differences in effect size. Correcting for measurement error, SOC accounted for approximately $25 \%$ of the variance in JS, and real differences in effect size accounted for less than $1 \%$ of the between-study variance.

\section{INTRODUCTION}

In a theory that would describe how people manage stress and stay well, Antonovsky's $(1979,1987)$ core construct was the sense of coherence (SOC), a dispositional orientation that is presumed to engender, sustain and enhance health, as well as to engender strength at other endpoints, such as work. It is a way-of-seeing-the-world which mitigates life stress by affecting the overall quality of stimuli that impact on the individual. The stimuli are perceived as comprehensible, i.e. making cognitive sense; as being manageable, under the individual's own control, as well as that of legitimate others (e.g. spouse, friends, formal authorities, political leaders, spiritual figures, but also systems such as a labour union, a medical aid programme or a pension fund); and as being motivationally meaningful, i.e. being welcome challenges worthy of engaging with and investing oneself in (Antonovsky, 1987, 1993). To someone with a weak SOC, the world could seem incomprehensible, hostile and absurd. SOC is presumed to be a product of idiosyncratic events in one's life, but also of having grown up in a particular social structure, culture and historical period (Antonovsky, 1987).

A working person with a strong SOC will make cognitive sense of the workplace and will perceive its stimuli as clear, ordered, structured, consistent and predictable information. Such a person will perceive work as holding challenges which she or he can meet by availing both personal resources and those under the control of, for example, managers, co-workers and subordinates. In addition, such a person will make motivational sense of work demands as challenges that are worthy of engaging with and investing personal energy in.

Job satisfaction (JS) remains a popular study area in industrial psychology, possibly because of the theoretical and practical attractiveness of a causal link between happiness on the job and improved job performance. Much research has focused on the relation between job conditions and JS, but recently there has been increased interest in the relations between dispositional variables and JS. Research shows that personality variables may influence JS: persons with stable positive core self-evaluations appear to be more satisfied with their jobs than people with negative core self-evaluations (Judge \& Hurst, 2007).

On theoretical grounds it seems likely that an individual with a strong SOC would experience high JS. Individuals high on SOC are likely to see the tasks put to them as challenges, to impose structure on the tasks and to search for appropriate resources (Antonovsky, 1987), which may enable them to find or create fulfilling and challenging job environments and to attract social support. In contrast, individuals low on SOC are more likely to see the tasks put to them as incomprehensible and potential sources of failure, which may lead them to become unmotivated, to gravitate to repetitive and uninspiring job environments, and to become alienated.

However, the relationship between SOC and work is likely to be bi-directional and work experiences could also, over time, strengthen or weaken the SOC, since it is not a fixed quantity. Long-lasting, severe and unpredictable stressors could produce experiences that negate one or more of the components of the SOC. When work experiences seldom call on one's abilities to actualise potential, one is unlikely to have confidence that the world is manageable; and incomprehensible, hostile experiences in a work context could undermine comprehensibility.

Antonovsky (1987) published a 29-item measure, referred to as the SOC-29, under the title of Orientation to Life Questionnaire. A 13-item short form, the SOC-13, was also indicated there. The SOC scale has been used in research in the world of work, mainly in the context of job stress (e.g. Albertsen, Nielsen \& Borg, 2001; Feldt, 1997; Feldt, Kinnunen \& Mauno, 2000; Kalimo, Pahkin, Mutanen \& Toppinen-Tanner, 2003; Kivimäki, Kalimo, \& Toppinen, 1998; Shiu, 1998; Söderfeldt, Söderfeldt, Ohlson, Theorell \& Jones, 2000).

The theoretical link between SOC and JS has received relatively little research attention. A quantitative synthesis of the results of several completed studies will give a more accurate picture of the relationship than any individual study. Moreover, such a synthesis will allow for the examination of whether the form of the SOC (SOC-29 vs SOC-13) moderates the strength of the relation between the two variables. Against this background we examined the strength of the relation between SOC and JS by performing metaanalyses on South African data. We hypothesised (i) that SOC is positively correlated with JS, (ii) that the form of the SOC (SOC-29 vs SOC-13) moderates the strength of the relation between SOC and JS, and (iii) 
that the type of JS measure (global vs composite) moderates the strength of this relationship.

\section{Research approach}

\section{RESEARCH DESIGN}

This study is a quantitative meta-analysis with the aim of synthesising the results of South African research on the relation between SOC and JS

\section{Research method}

The most important sources of data were unpublished honours degree research reports, masters and doctoral theses and dissertations; known South African academics working in the area of SOC were contacted personally in this connection too. The (South African) Journal of Industrial Psychology was searched by hand for articles on SOC and JS, from 1987 (when the SOC scale was published) to date. The South African Journal of Psychology, searched by hand, only produced articles by Strümpfer et al. Sets of unpublished data were also included. A diversity of samples resulted from these sources. Ten sets of data using the SOC-13 and 23 that used the SOC-29 were found. (An appendix containing all the data collected, another listing the sources of data, and a third indicating the classification of JS forms, are available from the second author.)

A diversity of measures of JS appeared in the data pool. For present purposes, the classification was as follows, although the distinction was not always clear-cut. A 'global' measure refers to general satisfaction/dissatisfaction with the job and contained two or a small number of declarative statements; the items were variations in wording of the same idea. A 'global general' measure is a variant where items were single adjectives or short phrases from which a variable number of items could be chosen by a respondent. 'Composite' measures consist of multiple items that provide, to a greater or lesser extent, coverage of various facets of satisfaction with different aspects of the job; in the present case, the number of items per scale varied between five and 100.

\section{RESULTS}

Meta-analysis was conducted with the Comprehensive MetaAnalysis Version 2 software (Borenstein, Hedges, Higgins \& Rothstein, 2006). Using a random effects model, the overall weighted mean correlation between SOC and JS across the 33 studies - with a total N of 3643 - was 0.42 (95\% CI: $0.38 ; 0.45)$. Across the full set of studies the null hypothesis of a homogenous effect size was rejected $\left(Q_{w}=54.54, d f=32, p=0.008\right)$. Real differences in effect sizes accounted for approximately $41 \%$ of the variance in the observed correlations. We tested for the potential moderating effect of the long form of the SOC (SOC$29 ; \mathrm{K}=23, \mathrm{~N}=2261$ ) versus the short form (SOC-13; $\mathrm{K}=10, \mathrm{~N}$ = 1382). For this analysis we employed a mixed effects model: a random effects model was used to combine studies within each of the two subgroups (SOC-13 vs SOC-29) and a fixed effects model was used to combine the two subgroups. This analysis revealed the correlation of 0.12 reported by Segaloe (1991) to be an outlier (this study had 197 participants, which makes it the largest study in the SOC-13 set and the third largest in the combined set). Upon removal of this study the weighted mean correlation between SOC and JS for the revised SOC-13 set ( $\mathrm{K}=$ $9, \mathrm{~N}=1185)$ was 0.41 (95\% CI: $0.35 ; 0.46)$, whereas the weighted mean correlation for the SOC-29 set was 0.43 (95\% CI: $0.40 ; 0.47)$. Within neither of the sets of studies could the null hypothesis of a homogenous effect size be rejected (SOC-13: $Q_{W}=5.17, \mathrm{df}=8$, $\mathrm{p}=0.739$; and SOC-29: $\left.\mathrm{Q}_{W}=28.81, \mathrm{df}=22, \mathrm{p}=0.150\right)$. Moreover the hypothesis of a homogenous effect size between groups could also not be rejected $\left(Q_{B}=0.76, d f=1, p=0.384\right)$.

We also tested for the moderating effect of the type of JS questionnaire. Here six studies were coded as using a global measure, whereas 25 were coded as using a composite measure.
Two studies that used the Job in General Scale (Ironson, Smith, Brannick, Gibson \& Paul, 1989) could not be combined with the global or composite measures and were excluded from the analysis. The weighted mean correlation for the composite measures ( $K=25, N=2629)$ was 0.42 (95\% CI: 0.39 ; 0.45), whereas the weighted mean correlation for the global measures $(K=6, N$ $=667)$ was 0.45 (95\% CI: 0.39; 0.52). Within both sets of studies the null hypothesis of a homogenous effect size could not be rejected (composite: $Q_{W}=27.114, d f=24, p=0.298$; and global: $\mathrm{Q}_{\mathrm{W}}=4.87, \mathrm{df}=5, \mathrm{p}=0.432$ ). Moreover, the null hypothesis of a homogenous effect size between groups could also not be rejected $\left(Q_{B}=0.73, \mathrm{df}=1, \mathrm{p}=0.392\right)$.

Next we computed a weighted mean correlation across the combined set of 32 studies with a total $\mathrm{N}$ of 3446 (without the Segaloe [1991] study) ( $\mathrm{r}=0.42 ; 95 \% \mathrm{CI}$ : 0.39; 0.45). Hence, across the range of studies it appears that SOC accounts for approximately $18 \%$ of the variance in JS. The between-study variance was very small $\left(\tau^{2}=0.001\right)$, with approximately $10 \%$ of the observed variance between studies being due to real differences in effect size.

The meta-analysis was also performed using the psychometric meta-analysis methods of Hunter and Schmidt (2004). After correcting the observed correlations between SOC and JS for measurement error in both variables, less than $1 \%$ of the observed variance between studies was due to real differences in effect size. The true correlation between SOC and JS - after correcting for measurement error - was estimated to be 0.50 .

\section{DISCUSSION}

The results provide strong support for the hypothesis that SOC is positively related to JS. On average, the meta-analysis shows that scores on the SOC measures accounted for approximately $18 \%$ of the variance in JS measures, whereas the theoretical SOC construct accounted for approximately $25 \%$ of the variance in the theoretical JS construct. The strength of the relation is somewhat surprising, given that the SOC does not contain any explicit workrelated items. This result is consistent with previous studies that found that personality variables have an effect on job satisfaction (Judge, Locke, Durham \& Kluger, 1998). The results also show that the long and short forms of the SOC relate equivalently to JS. These findings add support to the suggestion that researchers who wish to use a total SOC score may use the short form, which is less than half the length of the long form, without much loss of information or accuracy. Although the type of JS measure did not appear to moderate the strength of the relation between SOC and JS, it was noticeable that the global measures of JS correlated slightly higher than the composite measures.

A large number of potentially interesting moderators of the relationship between SOC and JS were not examined in this study, including job conditions (e.g. workload, autonomy, salary and type of job), personality variables (e.g. conscientiousness, agreeableness and emotional stability) and background variables (e.g. gender, race and culture). These variables could be the focus of further studies.

A question remains: to what extent is the SOC-JS relationship bidirectionally causal or due to the role of third variables? The role of, for instance, involvement in culturally valued enterprises (Antonovsky, 1987), job decision latitude (e.g. Karasek et al., 1998; Karasek \& Theorell, 1990) and job security/insecurity (Feldt et al., 2000) could be considered in this context.

\section{REFERENCES}

Albertsen, K., Nielsen, M.L., \& Borg, V. (2001). The Danish psychosocial work environment and symptoms of stress: The main, mediating and moderating role of sense of coherence. Work E Stress, 15(3), 241-253.

Antonovsky, A. (1979). Health, stress, and coping: New perspectives on mental and physical well-being. San Francisco: Jossey-Bass. 
Antonovsky, A. (1987). Unraveling the mystery of health: How people manage stress and stay well. San Francisco: Jossey-Bass.

Antonovsky, A. (1993). The structure and properties of the Sense of Coherence Scale. Social Science and Medicine, 36(6), 726733.

Borenstein, M., Hedges, L., Higgins, J., \& Rothstein, H. (2006). Comprehensive meta-analysis Version 2 [Computer software]. Englewood: Biostat.

Feldt, T. (1997). The role of sense of coherence in well-being at work: Analysis of main and moderator effects. Work $\mathcal{E}$ Stress, 11(2), 134-147.

Feldt, T., Kinnunen, U., \& Mauno, S. (2000). A mediational model of sense of coherence in the work context: A one-year followup study. Journal of Organizational Behavior, 21(4), 461-476.

Hunter, J.L., \& Schmidt, F.L. (2004). Methods of meta-analysis: Correcting error and bias in research findings. Thousand Oaks: Sage.

Ironson, G.H., Smith, P.C., Brannick, M.T., Gibson, W.M., \& Paul, K.B. (1989). Construction of a Job in General Scale: A comparison of global, composite, and specific measures. Journal of Applied Psychology, 74(2), 193-200.

Judge, T.A., \& Hurst, C. (2007). The benefits and possible costs of positive core self-evaluations: A review and agenda for further research. In D. Nelson \& C. Cooper (Eds.), Positive organizational behaviour (pp. 159-174). London: Sage.

Judge, T.A., Locke, E.A., Durham, C.C., \& Kluger, A.N. (1998). Dispositional effects of job and life satisfaction: The role of core evaluations. Journal of Applied Psychology, 83(1), 17-34.

Kalimo, R., Pahkin, K., Mutanen, P., \& Toppinen-Tanner, S. (2003). Staying well or burning out at work: Work characteristics and personal resources as long-term predictors. Work $\mathcal{E}$ Stress, 17(2), 109-122.
Karasek, R., Brisson, C., Kawakami, N., Houtman, I., Bongers, P., \& Amick, B. (1998). The Job Content Questionnaire (JCQ): An instrument for internationally comparative assessments of psychological job characteristics. Journal of Occupational Health Psychology, 3(4), 322-355.

Karasek, R.A., \& Theorell, T. (1990). Healthy work, productivity, and the reconstruction of working life. New York: Basic Books.

Kivimäki, M., Kalimo, R., \& Toppinen, S. (1998). Sense of coherence as a modifier of occupational stress exposure, stress perception, and experienced strain: A study of industrial managers. Psychological Reports, 83(3 Pt 1), 971981.

Segaloe, S. (1991). Sense of coherence as a moderator in the relationship between stressors and their work-related consequences among Black public service personnel. Unpublished master's research project, University of Cape Town, Cape Town, South Africa.

Shiu, A. T.-Y. (1998). The significance of sense of coherence for the perceptions of task characteristics and stress during interruptions amongst a sample of public health nurses in Hong Kong: Implications for nursing management. Public Health Nursing, 15(4), 273-280.

Söderfeldt, M., Söderfeldt, B., Ohlson, C.-G., Theorell, T., \& Jones, I. (2000). The impact of sense of coherence and highdemand/low-control job environment on self-reported health, burnout and psychophysiological stress indicators. Work E Stress, 14(1), 1-15. 